\title{
Evaluación de la estabilidad de excavaciones mineras de pequeño diámetro mediante clasificaciones geomecánicas y análisis empíricos: el caso de la mina de San Juan, Ecuador
}

\section{Evaluation of the stability of small diameter mining excavations using geomechanical classifications and empirical analysis: the case of the San Juan mine, Ecuador}

\author{
J. Cuervas-Mons, L. Jordá-Bordehore, J. A. Nazareno y K. F. Escobar \\ Escuela Superior Politécnica del Litoral, ESPOL, Facultad de Ingeniería en Ciencias de la Tierra, Campus Gustavo Galindo Km 30,5 \\ Via Perimetral, P.O. Box 09-01-5863, Guayaquil, Ecuador \\ (e-mail: josegeologo93@gmail.com, ljorda@espol.edu.ec,janio.nazareno@gmail.com,kescobar@espol.edu.ec)
}

\begin{abstract}
Resumen: Desde los años setenta del pasado siglo se viene empleando con éxito en todo el mundo una metodología de análisis preliminar de estabilidad de huecos subterráneos basada en el uso de las clasificaciones geomecánicas, siendo las más empleadas el RMR de Bieniawski y el índice Q de Barton. Esta última es la que se empleará en este estudio. Se trata de una aproximación empírica que permite estimar, mediante la asignación de una puntuación, la calidad del macizo rocoso, con el objetivo de conocer su grado de estabilidad ante la ejecución de una excavación subterránea. En la presente investigación se aplica el índice Q al estudio de la estabilidad de galerías y filones de una pequeña mina de oro en el Cantón de Ponce Enríquez, Ecuador. En este trabajo se propone un nuevo gráfico empírico tomando para ello otros anteriores y los resultados de la evaluación de tal manera que pueda determinarse el factor de seguridad y el máximo vano estable que pueda abrirse en minas de este tipo. Una de las principales aportaciones del trabajo es la de validar el índice de Barton a huecos pequeños.
\end{abstract}

Palabras clave: Índice Q, minería artesanal, mina subterránea, máximo vano, oro.

Abstract: Rock mass classifications are used throughout the world since the 1970's. It is a methodology for the prefeasibility assessment of the stability of underground openings. The most used are the Bieniawski's RMR and the Barton's Q index. The latter is the one we have used in this study. It is an empirical approach that gives a numerical score to the rock mass quality to determine the degree of stability or reinforcement required. In this research, the Q index is applied to assess the stability of galleries and veins in a small gold mine in Canton Ponce Enriquez, Ecuador. A new empirical chart has been established using previous ones and considering the results of the study. This chart can be used to determine the safety factor and stable maximum span that can be opened in vein-mines of this type. One of the main contributions of the work is to validate the Barton index to small holes.

Keywords: Q index, artisanal mining, underground mine, maximum span, gold. 
La estabilidad de huecos subterráneos como túneles, minas, cavernas hidroeléctricas y cuevas puede evaluarse en una primera aproximación mediante las clasificaciones geomecánicas y, en particular, con el índice Q (Barton, 1976). Este tipo de análisis lleva empleándose internacionalmente desde los años setenta del pasado siglo, sin embargo, está orientado principalmente a túneles y huecos mineros y centrales hidroeléctricas de cierto tamańo. No se ha encontrado referencias a la evaluación de huecos de tan pequeñas dimensiones como son las galerías mineras de acceso en minas artesanales, ni en labores de excavación de filones de pequeñas dimensiones.

La mina de oro de San Juan es una labor minera de pequeña entidad situada en el distrito minero de Ponce Enríquez, localizado al suroeste de la provincia de Azuay en Ecuador (Fig. 1). La minería de oro en la región es de tipo artesanal. Los yacimientos de la zona fueron descubiertos por azar a principios de la década de los años ochenta del siglo pasado, tras los fenómenos del Niño, que provocaron grandes deslizamientos en la zona de los yacimientos primarios. A raíz de ello se originaron localmente depósitos auríferos muy enriquecidos que llevaron a localizar otros coluviones y aluviales y a su vez las vetas. Se inició a partir de entonces la explotación del distrito en 1985 , y continuando hasta la actualidad por or- ganizaciones de mineros artesanales, teniendo poca o casi ninguna representación de minería de pequeña a gran escala.

Las labores mineras y galerías investigadas no tienen sostenimiento, por tratarse de una mina de pequeña entidad con medios escasos. No hay hasta la realización del presente estudio ningún análisis técnico que se haya efectuado en la mina, ni empírico, ni numérico, ni de cuñas sobre la estabilidad de las galerías o labores mineras en el filón, que justifiquen técnicamente el hecho de que en la mina no haya sostenimiento. Se trata de una mina en la que a simple vista se aprecian muy pocas zonas inestables (Fig. 2). Únicamente el emboquille está fortificado con bloques de piedra pequeños y hormigón debido a que está excavado en un material eluvial y de roca muy meteorizada, con evidentes indicios de circulación de agua.

En este tipo de minas de pequeña sección en rocas de buena a muy buena calidad geomecánica, lo habitual es no poner ningún tipo de refuerzo y solamente en las zonas de mala calidad que se atraviesan o donde se producen "campanas», se recurre a una entibación de madera. Sin embargo, aunque sea «evidente» la calidad de la roca y se emplee un sostenimiento basado en el empirismo y que localmente funcione, es necesario llevar a cabo un estudio geomecánico

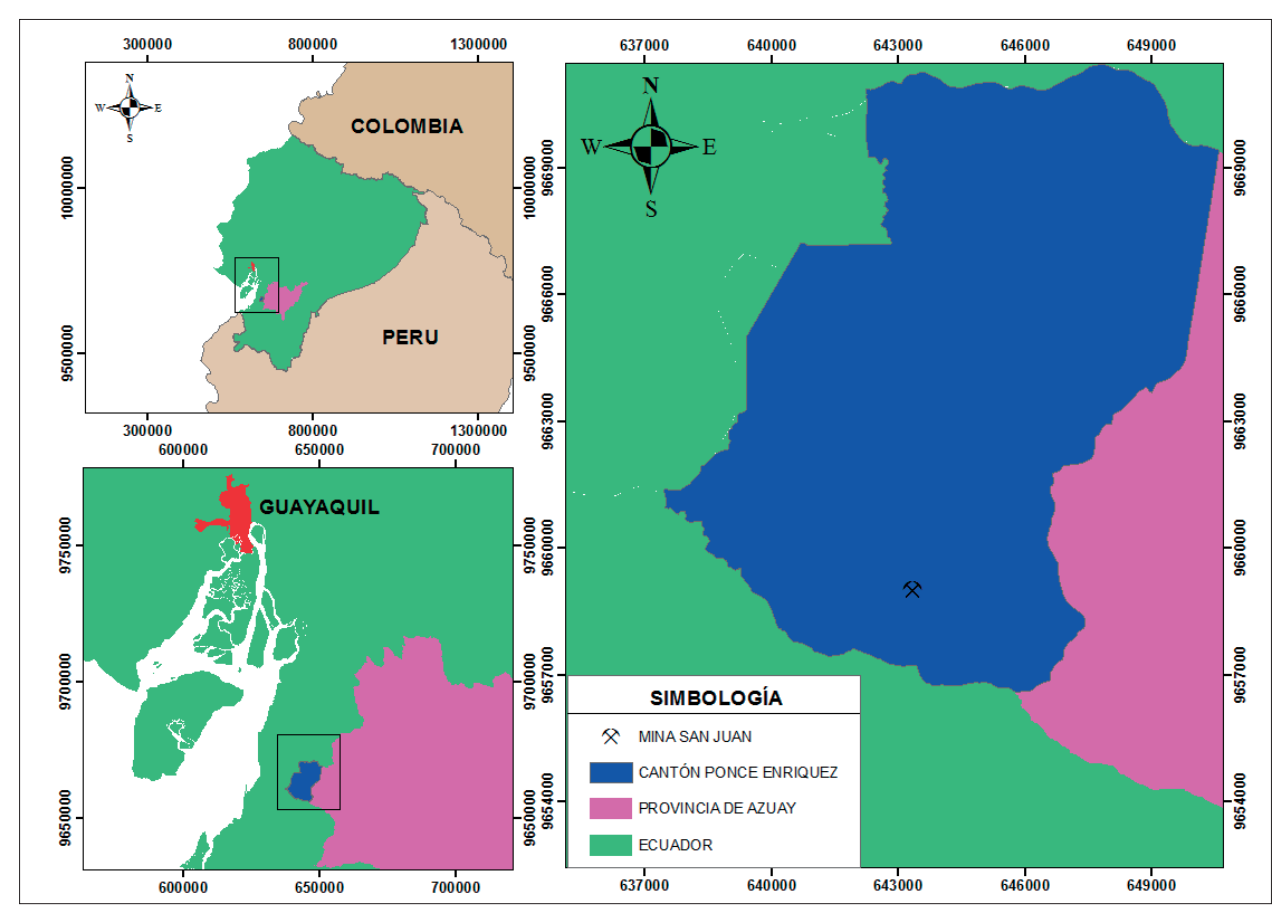

Figura 1. Situación geográfica de la mina San Juan, del Distrito de Ponce Enríquez y de la Provincia de Azuay, Ecuador. 


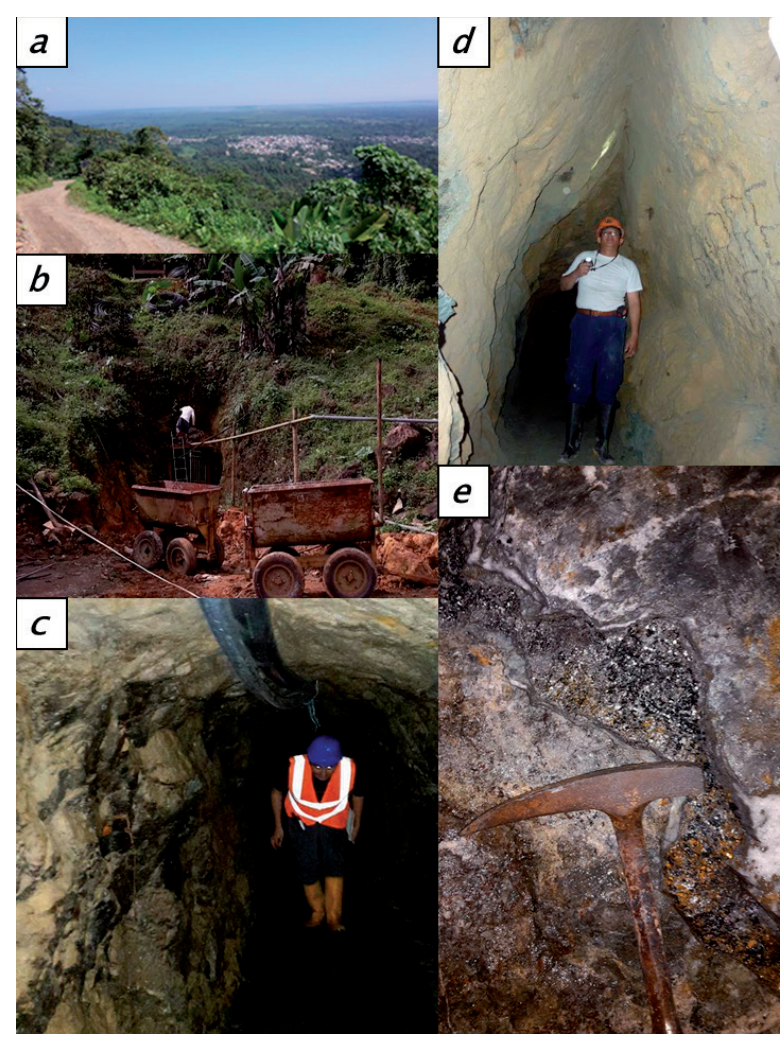

Figura 2. a) Panorámica de Ponce Enríquez, b) bocamina San Juan, $c$ ) galería de arrastre (nótese las reducidas dimensiones de la galería, las justas para poder empujar manualmente una vagoneta de ruedas), d) galería de arrastre en estéril de la mina San Juan (nótese la cuña caída y que la galería se ha estabilizado con ese «nuevo» ancho) y $e$ ) veta de sulfuros. Todas las fotografías fueron tomadas en el transcurso del año 2015. del macizo rocoso, para definir su grado de estabilidad ante la ejecución de una excavación subterránea.

Los objetivos del presente trabajo son determinar la calidad del macizo rocoso a partir de la clasificación geomecánica Q, a través del levantamiento de estaciones geomecánicas, y determinar la estabilidad de la excavación mediante el empleo de criterios empíricos, evaluando el vano máximo estable sin sostenimiento y el $\mathrm{D}$ equivalente.

\section{Contexto geológico y minero}

La mina estudiada se localiza dentro de la denominada Formación Macuchi del Paleoceno-Eoceno Medio (Cuervas-Mons, 2015). Esta formación comprende dos tipos de lavas andesítico-basálticas: unas andesitas porfídicas con fenocristales milimétricos de plagioclasas y unas andesitas basálticas de textura afanítica sin fenocristales. Puntualmente pueden aparecer serpentinitas de escaso espesor, tal y como se aprecia en una parte de la galería de arrastre de la mina estudiada (Fig. 3). Este sustrato rocoso se encuentra altamente fracturado por fallas y afectado por un conjunto de cuerpos granodioríticos, dioríticos y gabroicos de edad Plioceno-Mioceno, causantes del proceso metalogénico de la región (Cuervas-Mons, 2015).

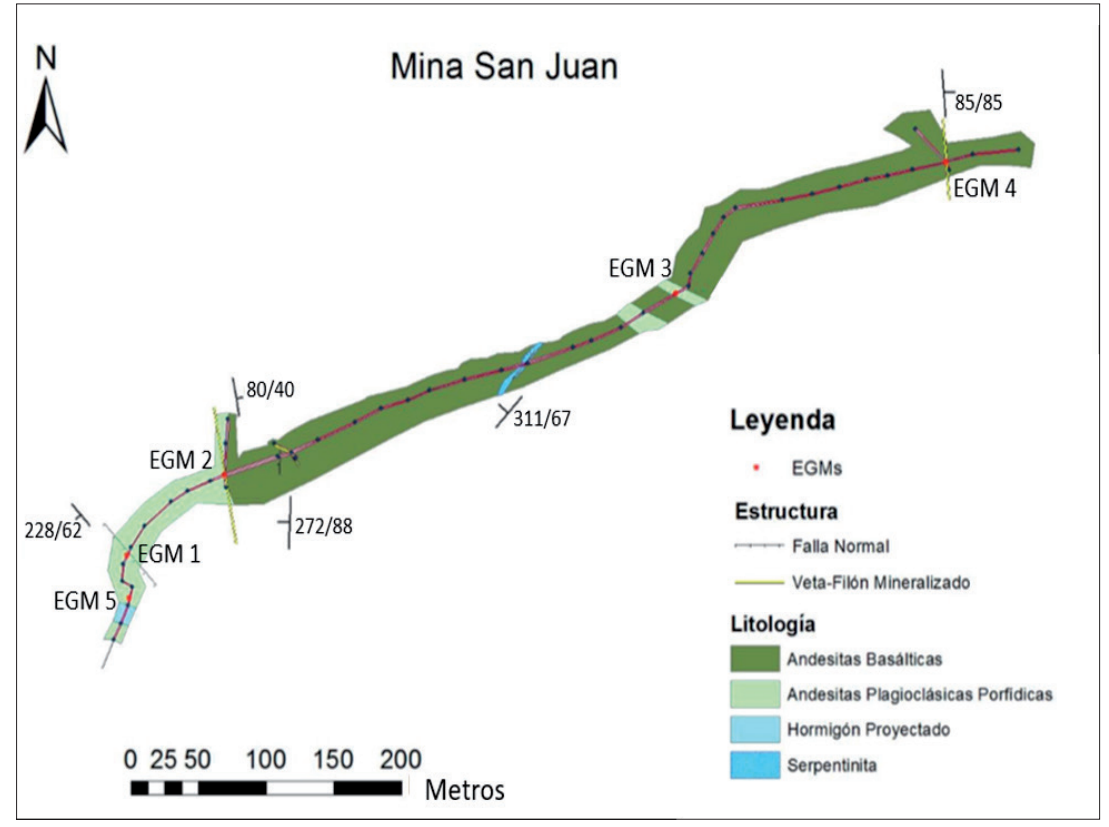

Figura 3. Plano geológico y de labores de la mina San Juan, donde se muestra la ubicación de las estaciones geomecánicas levantadas (modificado de Cuervas-Mons, 2015). 
Los niveles mineralizados con cuarzo, sulfuros y oro nativo tienen potencias centimétricas a métricas. Se agrupan en dos fajas estructurales de rumbos predominantemente $\mathrm{N} 75^{\circ} \mathrm{E}$ y $\mathrm{N} 15^{\circ} \mathrm{O}$, con un buzamiento de $30^{\circ}$ a $60^{\circ}$ hacia el este, estando tanto la mineralización como el diaclasado y la fracturación local y regional controlados por una serie de fallas con rumbo $\mathrm{N} 15^{\circ} \mathrm{O}$, también inclinadas hacia el este (Cuervas-Mons, 2015) (Fig. 3).

La explotación centra su interés económico en la extracción de Au y Ag, siendo la capacidad actual de explotación de la mina de 0,5 t/día, con leyes medias de entre 18 a $30 \mathrm{Au}$ g/t (Cuervas-Mons, 2015).

A fecha de realización del estudio en 2015 , la mina estaba en estado de preparación de labores. La mina consta de una galería principal en estéril, a modo de galería de arrastre, con una longitud aproximada de $900 \mathrm{~m}$ (Fig. 3). Esta galería va cortando los filones mineralizados, dos de las cuales ya habían sido reconocidos y parcialmente minados. El método de laboreo es el de realces y vaciado del filón, quedando autosostenido, dada la buena calidad del macizo y el pequeño ancho de los trabajos (máximo de $4 \mathrm{~m}$ ).

\section{Metodología}

Dado que se trataba de un trabajo preliminar y que en esta área no se disponía de estudios y los medios eran escasos, se optó por una metodología de análisis basada en la realización de estaciones geomecánicas, determinando in situ diversos parámetros geomecánicos: resistencia a compresión simple mediante esclerómetro y martillo de geólogo, rugosidad JRC con peine de Barton, etc. Se realizaron estaciones geomecánicas en las zonas de la mina donde había cambios litológicos o estructurales o bien en los cruces con los filones (Fig. 3).

Para evaluar la estabilidad del macizo rocoso, se ha recurrido al empleo de las clasificaciones geomecánicas y al análisis empírico. Esta es la metodología seguida desde los años setenta del pasado siglo en el prediseño de excavaciones subterráneas, internacionalmente admitida (Barton y Grimstad, 2004; Barton y Bieniawski 2008). Las clasificaciones geomecánicas más utilizadas son el índice Q (Barton et al.,
1974) y el Rock Mass Rating o RMR (Bieniawski, 1973, 1974, 1989, 2011).

El Sistema-Q, también llamado Q de Barton, fue desarrollado en Noruega en 1974 por Barton, Lien y Lunde, pertenecientes al Instituto Geotécnico Noruego. Se emplea para determinar las necesidades o cuantía de refuerzo de un túnel según su tamaño y la calidad de la roca. El índice $Q$ asigna a cada terreno un valor tanto mayor cuanto mejor es la calidad de la roca. Su variación no es lineal como la del RMR, sino exponencial, y oscila entre $Q=0,001$ para macizos muy malos y $\mathrm{Q}=1000$ para muy buenos.

El valor de Q se obtiene a partir de la siguiente expresión:

$$
\mathrm{Q}=\frac{\mathrm{RQD}}{\mathrm{Jn}} \times \frac{\mathrm{Jr}}{\mathrm{Ja}} \times \frac{\mathrm{Jw}}{\mathrm{SRF}}
$$

donde RQD es el índice Rock Quality Designation, es decir, la relación en tanto por ciento entre la suma de longitudes de testigo de un sondeo mayores de $10 \mathrm{~cm}$ y la longitud total. Jn varía entre 0,5 y 20 , y depende del número de familias de diaclasas que hay en el macizo. Jr varía entre 1 y 4 y depende de la rugosidad de las diaclasas. Ja varía entre 0,75 y 20 y depende del grado de alteración de las paredes de las diaclasas. Jw varía entre 0,05 y 1 dependiendo de la presencia de agua en el túnel. SRF es el Stress Reduction Factor y depende del estado tensional de la roca que atraviesa el túnel. Para la obtención de cada uno de los parámetros anteriores, se remite al lector a la extensa bibliografía al respecto y Tablas de aplicación (por ejemplo, Barton et al., 1974; Barton y Grimstad, 2004; NGI, 2013).

Para la estimación de los sostenimientos a aplicar en una excavación se emplea un gráfico de dos entradas (Fig. 4) donde en abscisas se introduce el valor de calidad Q del macizo rocoso y en ordenadas el ancho de la galería, túnel o labor minera. Sin embargo, ese valor de ancho o vano se modifica aplicando un factor de corrección para obtener un valor llamado De o Diámetro Equivalente del túnel, según la siguiente expresión:

$$
\mathrm{De}=\frac{\text { anchura diámetro o altura }(\mathrm{m})}{\mathrm{ESR}} \text { (2). }
$$


El ESR o Excavation Support Ratio, es un factor dependiente del tipo de excavación. Se estima a partir de la Tabla I. En el caso que nos ocupa se tomará un valor de ESR de 1,6 para la galería de arrastre y para las labores de vaciado del filón.
La figura 4 muestra 9 zonas a cada una de las cuales le corresponde un sostenimiento basado en cerchas, gunita y bulones (Grimstad y Barton 1993; NGI 2013). Así, por ejemplo, si tuviéramos una calidad de sostenimiento de $\mathrm{Q}=1$ y un $\mathrm{De}=7 \mathrm{~m}$, entonces

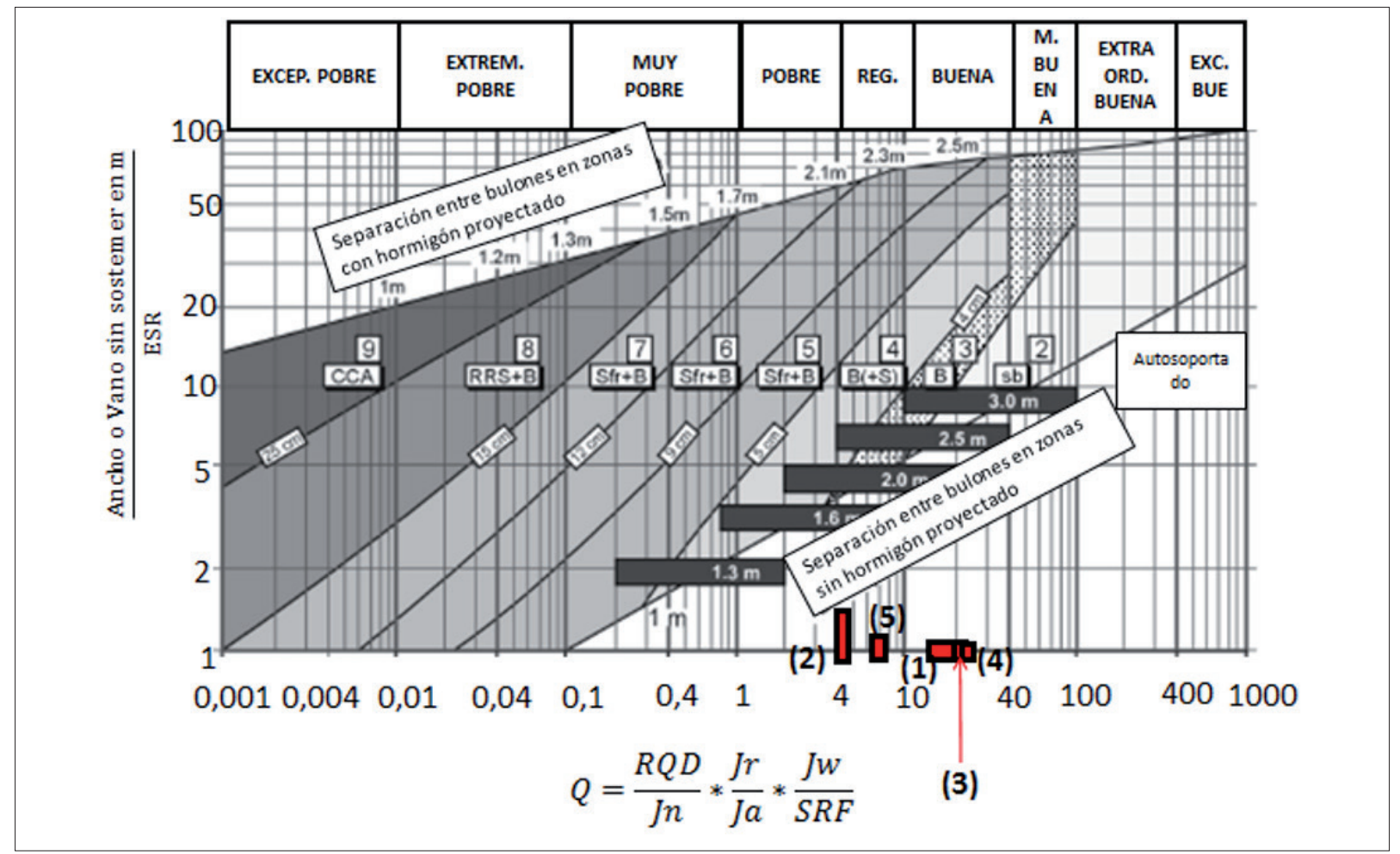

Figura 4. Estabilidad de las galerías estudiadas tomando las recomendaciones empíricas de túneles, determinada empleando el ábaco para estimar los distintos sostenimientos posibles a partir de la relación D equivalente e índice Q (Grimstad y Barton, 1993).

\begin{tabular}{|c|l|c|}
\hline \multicolumn{2}{|l|}{ Tipo de Excavación } & ESR \\
\hline A & Labores mineras de carácter temporal & $3-5$ \\
\hline B & $\begin{array}{l}\text { Pozos verticales: } \\
\text { - Sección circular } \\
\text { - Sección rectangular/cuadrada }\end{array}$ & 2,5 \\
2 & 1,6 \\
\hline C & $\begin{array}{l}\text { Huecos mineros permanentes, túneles de centrales hidroeléctricas, de suministro de agua, túneles piloto, galerías de } \\
\text { avance en grandes excavaciones }\end{array}$ & 1,3 \\
\hline D & Túneles menores de carretera y ferrocarril, chimeneas de equilibrio, túneles de acceso, colectores & 1 \\
\hline E & $\begin{array}{l}\text { Centrales eléctricas subterráneas, cámaras de almacenamiento, plantas de tratamiento de aguas, túneles importantes } \\
\text { de carreteras primarias y de ferrocarril, refugios subterráneos para defensa civil, emboquilles e intersecciones de } \\
\text { túneles }\end{array}$ & 0,8 \\
\hline F & Centrales nucleares subterráneas, estaciones de ferrocarril, instalaciones públicas y deportivas, fábricas & 0,5 \\
\hline G & $\begin{array}{l}\text { Cavernas muy importantes y huecos subterráneos con una duración de vida larga, 100 ańos o sin acceso para } \\
\text { mantenimiento }\end{array}$ \\
\hline
\end{tabular}

Tabla I. Valores del índice ESR (traducido de NGI, 2013). 
estaríamos en la zona 5 , donde se propone un refuerzo con gunita de 5 a $9 \mathrm{~cm}$ de espesor y bulones con un espaciado de $1,7 \mathrm{~m}$. Si por el contrario tuviéramos esa misma calidad o superior, pero con un diámetro equivalente de 1,5 m estaríamos en la zona 1 autosoportado, es decir, no se requeriría sostenimiento (Fig. 4).

La figura 4 es de aplicación principalmente a túneles y minas de cierta anchura y labores de cierta entidad que requieren diversos tipos y dimensiones de sostenimientos: bulones, cuadros metálicos, gunita, etc. En el caso de las galerías mineras y, en especial, de las excavaciones de pequeña sección (vanos de menos de 3 m) normalmente solo se consideran dos situaciones: roca de media a buena calidad en la cual no es preciso sostenimiento y una calidad por debajo de la cual se precisa un refuerzo. En las minas más artesanales ese refuerzo suele ser la entibación de madera mediante cuadros o puntales-estemples. Resulta, por tanto, fundamental conocer la calidad y el vano a partir de los cuales se considera que la mina no necesita sostenimiento. En los trabajos de Barton (1976) y Bieniawski (1989) se discute la aplicación del sistema Q para estimar la calidad de roca óptima para la estabilidad de dimensiones de túneles y cavernas hidroeléctricas sin sostenimiento. Un aspecto interesante del sistema Q es la posibilidad de reconocer las características del macizo rocoso que se requieren para la operación segura de huecos permanentes sin sostenimiento (Bieniawski, 1989). Después de analizar varios casos reales y de representarlo en un gráfico apropiado (Fig. 5) se obtienen los requisitos generales para huecos permanentes sin sostenimiento (Tabla II).

En la figura 5a los círculos representan excavaciones hechas por el hombre y los cuadrados cavernas naturales, de las cuevas de Carlsbad. La envolvente curva es una estimación del máximo vano de diseño para excavaciones hechas por el hombre sin sostenimiento y de modo permanente. Si lo combinamos con las cuevas naturales, la línea envolvente es aproximada-

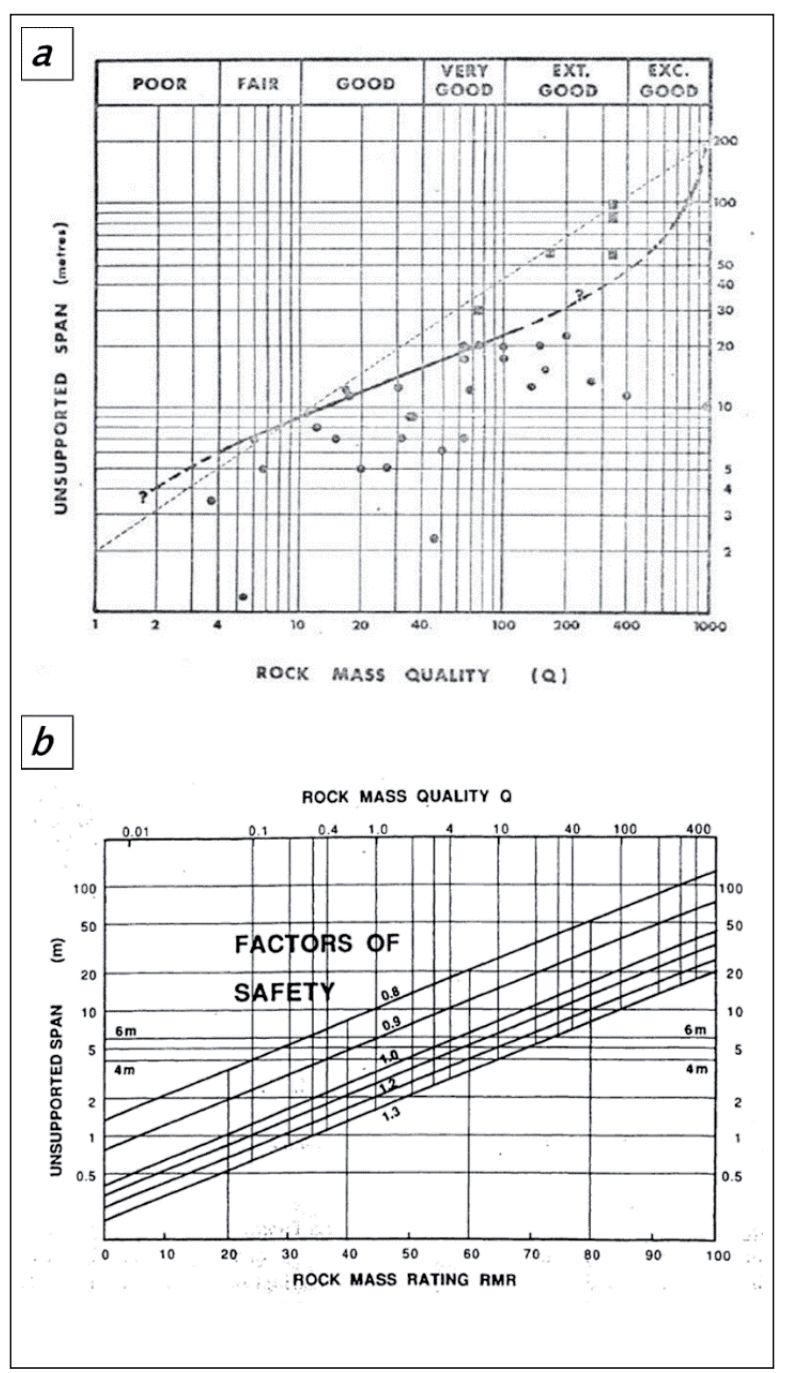

Figura 5. a) Vano de la excavación en ordenadas y calidad de la roca en índice Q en abscisas (Barton, 1976). b) Factor de seguridad estimado para excavaciones subterráneas sin sostenimiento en función del vano y la calidad de la roca (Bieniawski 1989, adaptado a su vez de Houghton y Stacey, 1980).

\begin{tabular}{|c|c|c|c|c|c|}
\hline $\begin{array}{l}\text { Requisitos } \\
\text { generales }\end{array}$ & $\mathrm{Jn} \leq 9$ & $\mathrm{Jr} \geq 1$ & $\mathrm{Ja} \leq 1$ & $\mathrm{Jw}=1$ & $\mathrm{SRF} \leq 2,5$ \\
\hline $\begin{array}{l}\text { Requisitos } \\
\text { condicionales }\end{array}$ & $\begin{array}{l}\mathrm{Si} R Q D \leq 40, \\
\text { entonces } \mathrm{Jn} \leq 2\end{array}$ & $\begin{array}{c}\text { Si Jn }=9, \\
\text { entonces } \mathrm{Jr} \geq 1,5 \mathrm{y} \\
\mathrm{RQD} \geq 90\end{array}$ & $\begin{array}{c}\text { Si Jr }=1 \text {, entonces } \\
\text { Jn }<4\end{array}$ & $\begin{array}{c}\text { Si SRF }>1, \\
\text { entonces } \mathrm{Jr} \geq 1,5\end{array}$ & $\begin{array}{c}\text { Si el vano es }>10 \mathrm{~m}, \\
\text { entonces } \mathrm{Jn}<9 . \\
\text { Si el vano es }>20 \\
\mathrm{~m} \text {, entonces } \mathrm{Jn} \leq 4 \mathrm{y} \\
\text { SRF } \leq 1\end{array}$ \\
\hline
\end{tabular}

Tabla II. Requisitos generales y condicionales requeridos para aperturas permanentes sin sostenimiento (Barton, 1976). 
mente lineal y puede representarse por la siguiente ecuación (Barton, 1976):

$$
\text { Vano }=2 Q^{0,66}
$$

Además de estas observaciones resulta interesante la figura 5b, que muestra un análisis cuantitativo para el factor de seguridad de excavaciones sin sostenimiento (Houghton y Stacey, 1980). Estos autores sugieren que, dados los diferentes propósitos de las excavaciones con aplicaciones civiles y mineras, se recomienda un factor de seguridad mayor de 1,2 cuando en una excavación se considere omitir el sostenimiento.

Para tomar los datos necesarios a fin de evaluar la estabilidad de las galerías se aplicará una metodología de campo basada en las estaciones geomecánicas. Se establecerán tantas estaciones geomecánicas como zonas geomecánicas diferenciadas o tipologías constructivas (sostenimientos, etc.) se reconozcan (Jordá et al., 2016).

\section{Resultados}

Durante el levantamiento de las estaciones geomecánicas se han utilizado las clasificaciones de RMR e índice Q. En el caso que nos ocupa es el índice Q el que presenta mayor interés, pues junto con el vano o ancho de excavación y el ESR, nos permite conocer el grado de estabilidad y el tipo de sostenimiento necesario de una excavación.

Basándonos en los datos obtenidos en las estaciones geomecánicas, los valores de cada uno de los parámetros o factores empleados para el índice Q se resumen en la Tabla III.

En primer lugar y antes de representar los pares de valores calidad-diámetro que representan los puntos de la Tabla III, se han unificado las figuras 5a y $5 \mathrm{~b}$ en la figura 6 . En esta figura se han añadido dos colores, uno verde para la zona estable, con factores de seguridad por encima de 1 y una zona inestable, que se ha representado en tono rojo. Por tanto, se tiene en cuenta el vano o ancho de la excavación (en metros), el índice Q y el factor de seguridad (Fig. 6). Este ábaco se utilizará para analizar la estabilidad de la mina en función de dichos parámetros. A partir de los valores calculados del índice $\mathrm{Q}$ y de las dimensiones de la excavación se obtiene el grado de estabilidad o de autosoportabilidad para cada una de las estaciones levantadas. La importancia de este ábaco reside en conocer el límite de la zona autoestable de las zonas que necesitan refuerzo (inestable en el gráfico).

\begin{tabular}{|c|c|c|c|c|c|}
\hline $\begin{array}{c}\text { EGM } \\
\text { (estaciones geomecánicas) \# }\end{array}$ & $\# 1$ & $\# 2$ & \#3 & $\# 4$ & $\# 5$ \\
\hline RQD (\%) & 91 & 77,25 & 93,85 & 93,85 & 77,25 \\
\hline Jn (diaclasas volumétricas) & 12 & 18 & 9 & 12 & 9 \\
\hline Jr (rugosidad diaclasas) & 3 & 2 & 2 & 3 & 2 \\
\hline Ja (alteración diaclasas) & 1 & 2 & 1 & 1 & 2 \\
\hline Jw (presencia de agua) & $0,66-1$ & 1 & 1 & 1 & 1 \\
\hline SRF (tensiones confinantes) & 1 & 1 & 1 & 1 & 1 \\
\hline Q & $15,02-22,75$ & 4,3 & 20,9 & 23,46 & 8,6 \\
\hline Ancho de excavación & 1,2 & $1,94-2,21$ & $1,35-1,6$ & 1,3 & 1,05 \\
\hline ESR & 1,6 & 1,6 & 1,6 & 1,6 & 1,6 \\
\hline Diámetro equivalente & -1 & $1,2-1,4$ & -1 & $\sim 1$ & -1 \\
\hline
\end{tabular}

Tabla III. Valores de los parámetros geotécnicos para el cálculo del índice Qy la calidad de macizo rocoso, así como los anchos de la excavación, el ESR y el Dequiv. para cada una de las estaciones geomecánicas. 


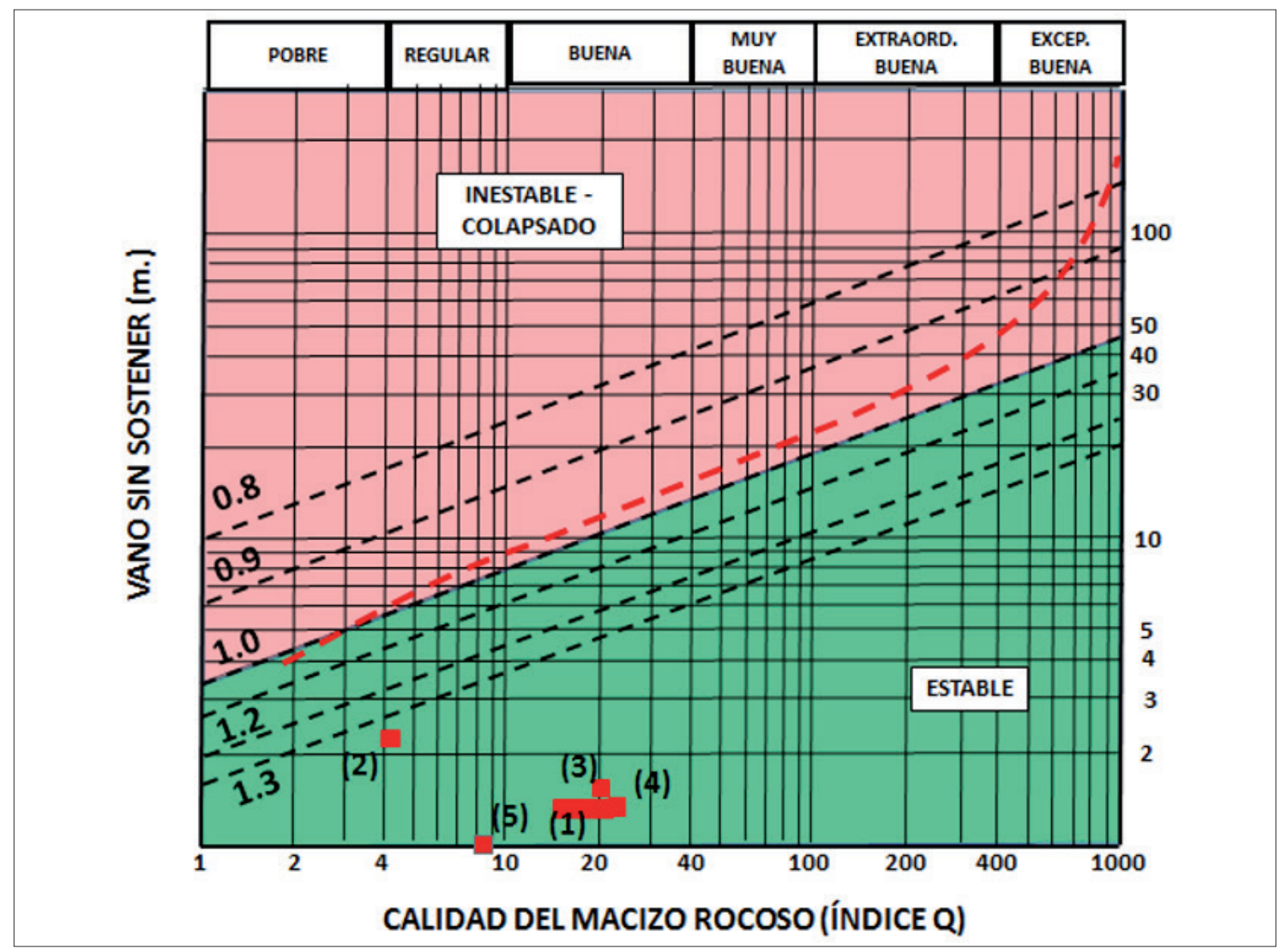

Figura 6. Gráfico para estimar la estabilidad de la excavación a partir del índice Q de Barton, el vano sin sostener (m) y el factor de seguridad. Los números entre paréntesis corresponden a las estaciones geomecánicas levantadas.

En la figura 6 han sido representados los puntos de la Tabla III. Como se aprecia, las 5 zonas de la mina estudiadas y que son estables, aparecen en la zona estable. La calidad es relativamente buena y el ancho de la galería o labores muy pequeña. El factor de seguridad en todas ellas es alto, por encima de 1,3. Sin embargo si tomamos por ejemplo el punto 3 que corresponde a una calidad de roca $\mathrm{Q}=20,9$ y un ancho menor de 1,6 m en vano, vemos que aumentando su anchura (ascendiendo en ordenadas), entonces la estabilidad disminuye. Para un vano en torno a 5 el factor de seguridad sería ya estrictamente de 1,3 y a partir de $10 \mathrm{~m}$ la galería sería inestable. Por tanto, en esta zona de la mina no podríamos abrir vanos de más de $10 \mathrm{~m}$ sin reforzar el macizo.

Como se puede comprobar en la figura 6 , todas las estaciones geomecánicas se encuentran dentro del área de estabilidad, $\mathrm{y}$, por tanto, la mina en toda su extensión sería autosoportable. El factor determinante de la estabilidad para la explotación es el vano o ancho máximo excavado.

Este gráfico de autosoporte de la figura 6 es en este caso más útil y sencillo de aplicar que aquellos de los túneles (Fig. 4). Sin embargo, se puede realizar el ejercicio de introducir los puntos de la Tabla III en la figura 4. Analizando esta figura, se puede comprobar nuevamente que todas las estaciones geomecánicas, y, por tanto, toda la mina en su extensión, se encuentra dentro del área de autosoportabilidad y, por lo tanto, no requiere ningún tipo de sostenimiento o entibado. Los vanos son pequeños y una vez escalados en De mediante el ESR quedan en aproximadamente un ancho de 1 m., desplazándonos sobre el propio eje de abscisas u ordenadas de la figura 4. Para estos anchos de galería deberíamos tener una calidad mucho me- 
nor $(\mathrm{Q}<0,1)$ para que se requiriese sostenimiento, encontrándonos en las zonas 5, 6, y 7 de refuerzo (según Grimstad y Barton, 1993). El sostenimiento que se recomendaría según lo establecido en esta figura 4 seria de hormigón proyectado con fibras y bulones separados de $1 \mathrm{a} 1,3 \mathrm{~m}$. A todas luces este sostenimiento no tiene ningún sentido en galerías de mina provisionales o en minería artesanal donde es inviable este tipo de actuaciones. En estos casos lo más frecuente es el uso de entibación de madera en aquellas zonas locales, donde fuese necesario.

\section{Conclusiones}

La mina San Juan es una explotación de oro de tipo artesanal excavada en rocas de naturaleza andesítico-basálticas. Las labores tanto de galería como los cruceros en filón son en general, estables, y solo se aprecian puntualmente caídas de cuñas de roca. Las dimensiones reducidas de la excavación permiten por tanto que las labores sean autosoportadas. Empleando los ábacos habituales del índice Q se ha determinado que el límite de la estabilidad estaría en un ancho o vano de entre 6 y $10 \mathrm{~m}$. De una forma totalmente observacional y «empíricamente tradicional», los mineros han llegado a la conclusión de que el macizo rocoso es de buena calidad y la mina puede ser autosoportada. Sin embargo y como resulta evidente, este tipo de aseveraciones no tienen ninguna base geomecánica que sea aceptada y aceptable desde el punto de vista ingenieril. Las clasificaciones geomecánicas y en particular en este caso el índice $Q$, es una metodología muy sencilla de aplicar y que permite establecer rangos de estabilidad y sostenimiento apoyados por datos empíricos reconocidos a nivel mundial. De forma muy económica y sencilla podemos dar una justificación adecuada y técnica a las aseveraciones antes apuntadas. Mediante estaciones geomecánicas realizadas en dos días de campo y medidas topográficas muy sencillas junto con el trabajo de gabinete de determinación del índice $\mathrm{Q}$, se ha podido justificar la no necesidad de sostenimiento. Pero, además, empleando los diferentes gráficos que relacionan anchos con calidad de roca, se puede extrapolar cuál es el máximo ancho que se le puede dar a una labor para tener un factor de seguridad determinado o dónde se encuentra el máximo de la autoestabilidad en vano-metro. Una de las principales aportaciones del trabajo es la de validar el índice de Barton a huecos pequeños.

\section{Agradecimientos}

Agradecemos a los propietarios de la Cooperativa 12 de Octubre, dueńos de la mina San Juan, y a su ingeniero supervisor Franklin Carrillo Cevallos el interés y ayuda prestada durante la realización de este estudio.

\section{Bibliografía}

Barton, N. (1976): Unsupported underground openings. Rock Mechanics Discussion Meeting, Stockholm: 61-94.

Barton, N. y Bieniawski, Z. T. (2008): RMR and $\mathrm{Q}$ : Setting records. Tunnels and Tunnelling International, 2008: 26-29.

Barton, N. y Grimstad, E. (2004): The Q system following thirty years of development and application in tunnelling projects. Eurorock 2004 53rd geomechanics Colloquium.

Barton, N., Lien, R. y Lunde, J. (1974): Engineering classification of rock masses for the design of tunnel support. Rock Mechanics, 6(4): 189-236.

Bieniawski, Z. T. (1973): Engineering Classification of jointed rock masses. Transactions of the South African Institution of Civil Engineering, 15: 335-344.

Bieniawski, Z. T. (1974): Geomechanics Classification of rock masses and its application in tunnelling. Advances in rock mechanics, 2(A): 27-32.

Bieniawski, Z. T. (1989): Engineering Rock Mass Classifications, Wiley, New York.

Bieniawski, Z. T. (2011): Errores en la aplicación de las clasificaciones geomecánicas y su corrección. Conferencia magistral Adif-Geocontrol, 35 p. 
Cuervas-Mons, J. (2015): Estudio geológico-estructural y análisis geomecánico en la Mina San Juan; Distrito Minero Ponce Enríquez, Provincia de Azuay, Ecuador. Proyecto Fin de Carrera, FICT-ESPOL, Ecuador.

Grimstad, E. y Barton, N. (1993): Updating of the Q-System fr NTM. Proceedings of the International Symposium on Sprayed Concrete-Modern Use of Wet Mix Sprayed Concrete for Underground Support, Fagernes, Oslo.

Houghton, D. A. y Stacey, T. R. (1980): Application of Probability Techniques to Underground
Excavation, Proceedings of the 7th Regional Conference for Africa on Soil Mechanics and Foundations of Engineering, Ed. A.A. Balkema, Accra, 2: 879-883

Jordá, L., Tomás, R., Arlandi, M. y Abellán, A. (2016): Manual de estaciones geomecánicas. Descripción de macizos rocosos en afloramientos. Ed. ETSI Minas. Grupo de Proyectos de Ingeniería, Universidad Politécnica de Madrid, Madrid, 200 p.

NGI (2013): Using the Q system. Handbook. Rock mass classification and support design. Ed. NGI, Oslo, $57 \mathrm{p}$. 